\title{
Emotishare: Emotion Sharing on Mobile Devices
}

\author{
Matthew John Willis \\ University of the Sunshine Coast, \\ Queensland, Australia. \\ mwillis@usc.edu.au
}

\author{
Christian Martyn Jones \\ University of the Sunshine Coast, \\ Queensland, Australia. \\ cmjones@usc.edu.au
}

\begin{abstract}
Emotishare is a web and mobile platform for users to continuously track, share and respond to the emotional states of their friends. The system was trialled with both large and small groups to explore emotional communication. The groups were provided with two alternate interfaces to the system (web and mobile), and usage was compared in order to determine the effectiveness of each interface in supporting emotional communication. While overall usage behaviour was unaffected across both systems, the results highlighted that the mobile system was better suited to encouraging ad-hoc emotional tracking, sharing and response behaviour.
\end{abstract}

Human-Computer Interaction, Affective Computing, Emotion Sharing, Mobile Technologies.

\section{INTRODUCTION}

Society is increasingly relying on technology for daily communication requirements. Email, forums, blogs and sites such as Twitter are popular methods of communication, and social networking sites are helping their users to manage the large number of social connections that are made everyday. Faster wireless networks and more powerful mobile devices are making the use of this technology more frequent and widespread.

While access to this technology has increased, the basic premise for composing and sending messages remains unchanged. Voice and short text-based messages are still in widespread use, and some devices offer emotional cues in the form of 'emoticons' to allow users to include some personality and expression of emotion in their messages. When emotional state is communicated in existing social networking systems, it is often presented alongside additional content (such as comments, media or links to other websites), which can alter the perception of the communicated state. While these cues do facilitate the communication of emotion (with respect to the communicated message), there does not yet exist an effective method of communicating one's current emotional state for the sole purpose of sharing that state.

\section{EMOTIONS AND EMOTION COMMUNICATION}

Emotions are communicated to others in our social network using various cues (such as facial expression, body language, vocal intonation and behaviour). It is the integration of these cues that results in an emotional communication (Planalp and Knie, 2002).

Communication that occurs without the transmission of emotion cues risks a loss of meaning. In this case, the interpretation of the message is left up to the receiver. The receiver must rely on their own contextual understanding and personal experience to decode the message received, which can result in a change to the intended meaning (Coupland et al., 1991).

This is not to say that communication without cues to emotion are unsuccessful, rather that the purpose of the message is important when considering the medium of the message. Planalp (1999, pp 39) suggests two theoretical perspectives on communication to distinguish this difference: Communication as exchanging messages, and communication in coordinating meaning. In the former, a text-based message sent from a mobile phone may be the most efficient and appropriate, and in the latter a face-to-face interaction may be more appropriate as it provides more opportunities to convey non-verbal cues to emotion.

\section{EMOTIONS AND TECHNOLOGY}

Support for communication through technology has increased dramatically over the past 10 years. Ubiquitous technologies (such as mobile phones, music players and other mobile gadgets) have become more powerful and provide always-on connections to the Internet, allowing users to 
maintain constant contact with family and friends. Voice communication using mobile phones has been supplemented with new services that allow users to communicate in new ways. Social networking platforms such as Facebook (2012) and Twitter (2012) encourage users to make and maintain a large number of connections with others and help users to share more information about every aspect of our lives.

While access to new social technologies has increased, the basic process of communicating using these platforms has remained unchanged. Short text-based messages still feature as the most common form of communication, with punctuation, spelling and emoticons taking the place of body language as cues to emotion expression (Riordan and Kreuz, 2010). The problem with these cues is that they are often misunderstood and can affect the intention and meaning of the communicated message (for example, see: Kato et al., 2007, Lo, 2008, Riordan and Kreuz, 2010). In addition, emotional cues (sent via short messages) are often used as a conscious expression of emotion directed at the content of the message rather than an expression of one's current emotional state.

Derks and colleagues (Derks et al., 2008) also highlight some of the issues with emotion expression in computer-mediated communications $(\mathrm{CMC})$, suggesting that the use of emoticons is 'deliberate and voluntary' (2008, pp 778) and that more complex forms of emotion communication (such as mimicry) 'cannot be fulfilled by the use of emoticons' (2008, pp 779).

While emoticons may be an effective way of representing an emotional state, the meaning associated with the state and the purpose of its inclusion in a message can be easily misunderstood. Current systems employ the use of emoticons as cues alongside large amounts of content, or as a cue tagged to another message. The system presented in this paper removes this extra content and provides a platform for users to share their current emotional state with each other, free from contextual distraction. In doing so, it has allowed for the exploration of emotion sharing between members of a social group using digital communication.

\section{THE EMOTISHARE SYSTEM}

The Emotishare system was designed to allow members of a social group to have access to the reported emotional states of others, and provide awareness of these states at all times. The research considered how this knowledge assists people to make decisions about responding to these emotional states, and how people choose to communicate with others (for more on the Emotishare research project, see: Willis, 2011).

The Emotishare system is a cloud-based web application that provides both a web browser and a native iPhone application as its interface. The system is comprised of the following components (see Figure 1).

- Emotion Report and Response Interfaces (web and mobile),

- Emotishare Cloud Service (provides the services that allow for the sharing of emotion states between devices)

- Data Storage (Provides a historical view of emotion states and relationships between users of the system).

In addition, the Emotishare system offers an Application Programming Interface (API) so that additional devices can utilise the system to both report and retrieve the emotional state of a user.

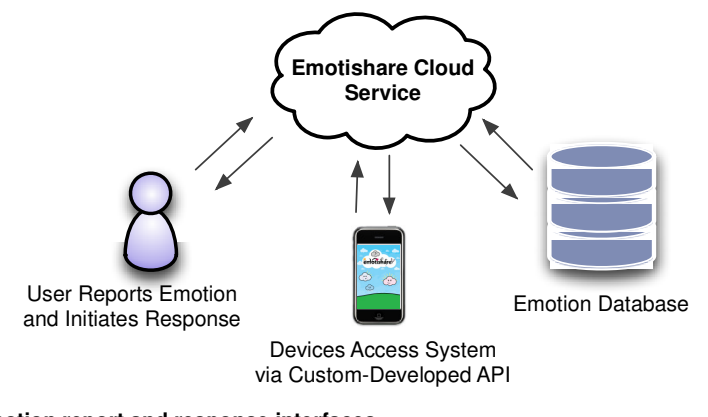

(Emotion report and response interfaces, web and mobile).

Cloud-based Storage

Figure 1: Emotishare System Diagram (Overview).

Emotishare was designed as a research tool to allow for the controlled tracking of emotion reports and responses between members of a social group. Functionality is intentionally restricted so not to distract the user with additional content that could obscure the emotion message. Emotishare allows for the formation of small intimate groups that are created for the purposes of sharing emotional state. Research has shown that intimate groups (Lickel et al., 2000) may be good candidates for the sharing of personal information (such as emotional state). The Emotishare system allows its users to control who can view and respond to their emotional state.

While existing social networking systems can provide similar services for the sharing of emotional cues, they do so by also presenting other media and content and allow access to a large group of people with whom the user may not be willing to share this information. In addition, existing social networking services encourage large group memberships (by prompting users to create more friendships and displaying total friends counts to 
other users). This can be problematic for supporting the sharing of information such as emotional state and may actively discourage such sharing to take place.

The current version of the Emotishare system allows users to report 10 discrete emotional states. Each state is represented as a 'cloud character', similar in representation to existing emoticons available in other systems. The cloud characters were tested with the participant groups before the trial to ensure they were recognised as representing the state intended. In addition, an emotion label was presented alongside each cloud character to minimise the potential for confusion in recognising the corresponding state. The states used were: 'Happy', 'Admiring', 'Scared', 'Surprised', 'Sad', 'Disgusted', 'Bored', 'Angry', 'Frustrated' and 'Interested' and are based on Plutchik's (1980) discrete model of emotions.

\subsection{Web Application}

The Emotishare web application is a secure web site that allows users to log on and see the emotional states of others. When a user logs into the system, they are presented with the user dashboard (see Figure 2), which provides access to all of the features of the web system in a single interface. Users can view the states of their friends (see Figure 2, highlighted as star 1), set their own emotional state (see highlight 2), view friend requests (see highlight 3 ), respond to a friends emotional state (see highlight 4), and request friendships from other Emotishare users (see highlights 5 and 6). The Emotishare system also provides users with support information (see highlight 7) and allows the user to log off to keep their information secure (see highlight 8).

\subsection{Mobile Application}

The mobile application provides users with a native iOS application that they can use on the Apple iPhone. The mobile system offers all of the functionality of the web-based system, with the addition of a text-based message to a friend request. The mobile application presents each feature available on its own tab (see Figure 3). The mobile application also provides direct access to the iPhone's functions (such as Phone, SMS and Email) to allow users to respond easily to others after viewing emotional states.

\section{EXPERIMENT DESIGN}

The Emotishare system was deployed to members of large social groups followed by another trial with smaller 'intimate' groups. The large group trial used the web version of the system, while the smaller groups alternated usage of both the web and mobile versions of the system. This paper presents results related to the usage patterns between both versions of the system, therefore only the results from the small group trial are presented.

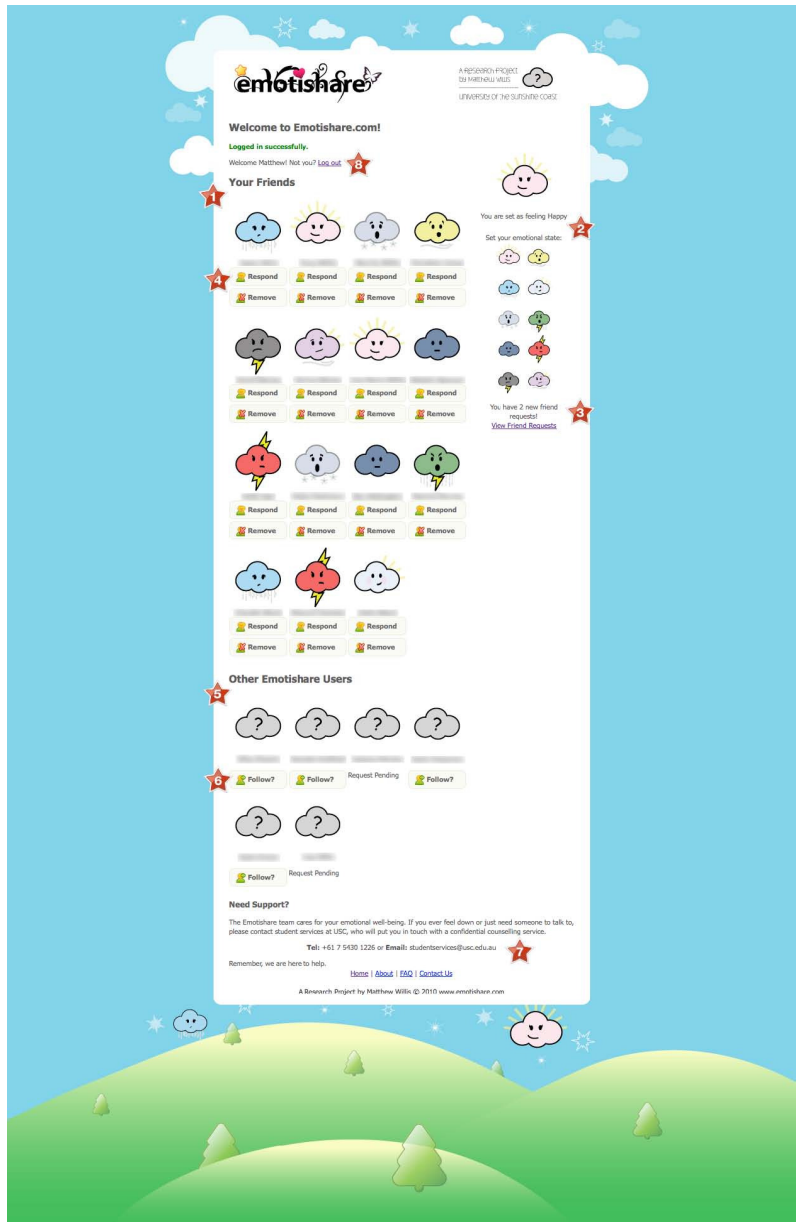

Figure 2: Emotishare System - User Dashboard (Web version. Highlights refer to discussion in main text. Participant names have been blurred for privacy).

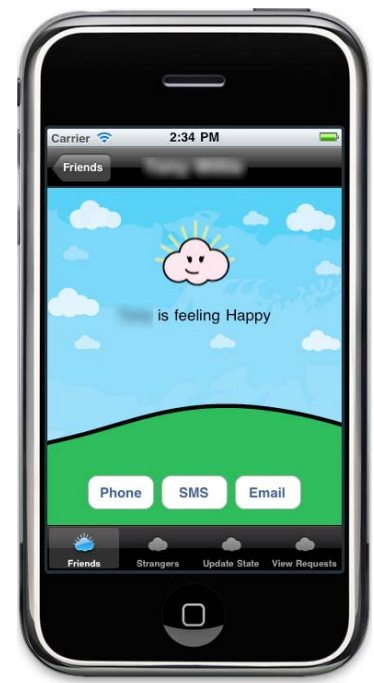

Figure 3: Emotishare System - Friends State Details Screen (iPhone version. Participant names have been blurred for privacy reasons). 
Intimate groups were identified as those social groups that contain strong ties between members (Lickel et al., 2000) and are defined hereas 'A close-knit social group, such as those containing friends and family'.

Three small groups participated in the research study. Participants were members of a Mother's Group (support groups designed for first time mothers) located in Sydney and the Central Coast in New South Wales, Australia. Each group contained less than 10 participants, met once per week (in person) and used social networking services (such as Twitter (2012) and Facebook (2012)) to stay in touch between meetings.

During the Emotishare system trial, two of the groups alternated between web and mobile system usage, while the third provided a control group (who did not use the system). The control group was surveyed for their general emotion sharing behaviour during the trial period. This data was compared with the same data collected from the trial participant groups to determine the impact of the Emotishare system on the emotion sharing behaviours of the participants. The system trial lasted for 5 weeks. 1 week was provided for mothers to gain familiarity with using an iPhone. The system was trialled with each group for a period of 2 weeks. Surveys were provided to participants at three stages during the trial to collect data on their experience using the system. Usage of the system was logged and stored in an online database. Finally, a focus group activity was carried out at the completion of the trial period. For an overview of the trial, refer to Figure 4.

\section{RESULTS}

Participants were surveyed during system use for their preferred general methods of communication and their preferred method when using the Emotishare system. Participants were also surveyed for their opinions of the Emotishare system, and its ability to support the communication of emotional state. Surveys took place at different intervals throughout the trial period. The pre-system usage survey investigated the existing communication habits of participants, the system survey tracked these habits over the trial period and the post-system usage survey investigated experiences of the participants with the Emotishare system.

In the pre-system usage survey, the top ranked communication method was SMS message via a mobile phone. The ranked order was 'Face-toFace', 'Telephone', 'Social Networking Sites' and 'Email'. 50\% of the participants reported spending between 30 minutes and 1 hour using online services per day to communicate with friends. When asked about communication of emotional state, participants ranked the telephone (SMS or voice call) and Email methods of communication higher than Social Networking Sites in order of preference of use.

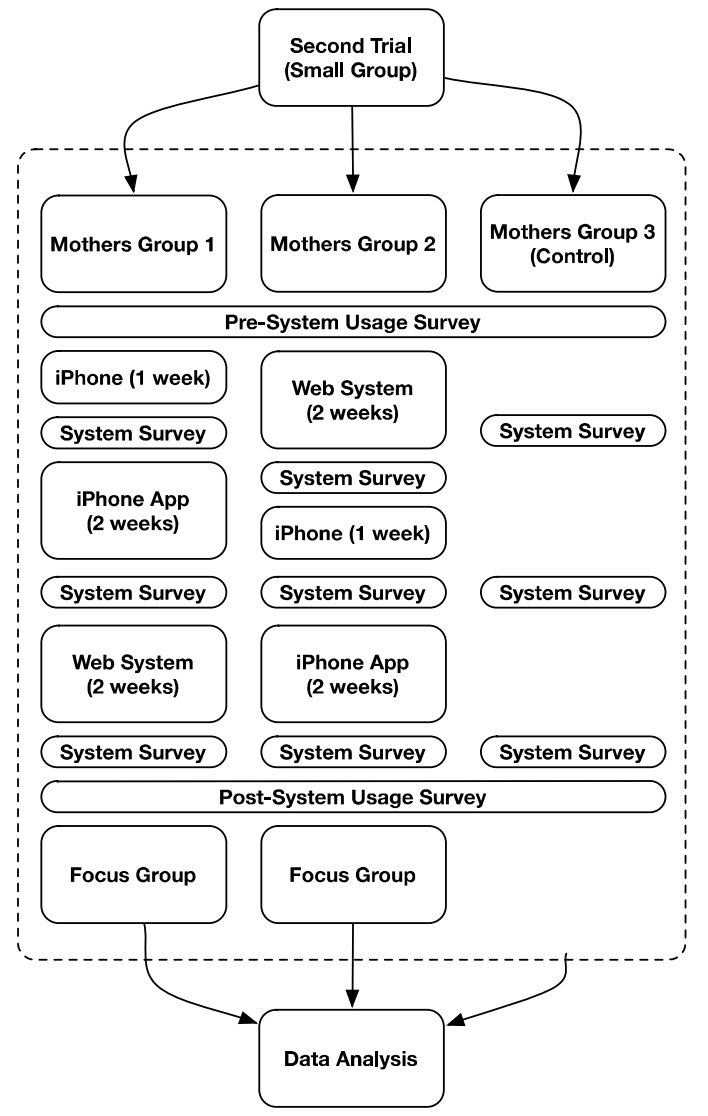

Figure 4: Experiment Design Overview (Small Group Trial).

System activity was recorded during usage. There were some slight functional differences between the web and mobile systems due to the platforms and usage requirements. For example, system features available on a single page in the web system were presented in a tabbed interface in the mobile system. While some minor functional differences are present, the data does offer an insight to usage patterns of each system. The web system accounted for $41.8 \%$ of all recorded activity while the mobile system accounted for $58.2 \%$. There was consistent usage behaviour between both platforms. The most commonly recorded activity for the web system was 'Checked Friend's States' (32.4\%) followed by 'Set Own State' $(24.1 \%)$. The mobile system showed the most commonly recorded activity to be 'Checked Friend's States' (46.2\%) followed by 'Checked Own State' (29.7\%).

The order of system usage also had an impact on the recorded usage. For the participant group that used the web system first, $56.7 \%$ of usage was attributed to the web system, followed by $43.2 \%$ for 
the mobile system. In contrast, the group that started with the mobile system showed $86.4 \%$ of usage for the mobile system with a large drop in usage for the web system.

The survey results revealed that participants felt they used the web system more often for reporting emotional state and the mobile system more often for checking on the emotion reports of others. During the focus group activity, the participants were questioned on their overall preference for each platform. One participant described their thoughts on the benefits of the mobile over the web system:

I think the phone [app] you are more likely to be truthful, because it's a lot more personal, you know that nobody else can see it, it's your device.

This comment suggests that the ubiquitous nature of the mobile technology had an impact on the perception of privacy of data shared using the Emotishare system. Even though the emotion data was shared with the same group of people, mothers felt more at ease sharing this type of information using their mobile phone than through a web page. In addition to feelings of privacy, participants also suggested that emotion state viewed in Emotishare might be perceived as being more truthful than on a medium with larger connections (such as Facebook (2012)). One participant suggested that:

If it were a bigger group, there's colleagues, or there's family that you mightn't want to tell everything, but in a group where we are generally going through similar emotions, you are more likely to be saying, you know, 'I've had a shocker' or... something personal... and be truthful about it.

Another participant agreed:

I would be more likely to go on Facebook and say I am fine when I am not, because I think its a big audience... whereas if I am on text message or Emotishare... I would be more honest.

Another participant suggested that the mobile system was beneficial for ad-hoc use:

\begin{abstract}
I didn't consciously think 'Oh, I need to go to the website and update how I am feeling', where on my phone, if I am flipping through my phone and then I saw it I was like 'Oh, I'll push that and see what's going on'... so the iPhone was much easier than the website.
\end{abstract}

There was a strong symmetry between the emotion states reported on both systems, with one exception. Reports of the 'Scared' emotion state were more frequently reported using the mobile version of the system. The platform did not have an effect on the response behaviours of participants using the system, and methods used to respond to an emotional state did not appear to be affected.

\section{DISCUSSION AND CONCLUSION}

This paper presents results on investigating the sharing of emotion data across different platforms (mobile and web). The medium used to access the Emotishare did not appear to impact on the emotions participants chose to respond to. In both systems, short messages sent via a mobile phone were the preferred method of response to an emotional state, however this did differ depending on the emotion that was being responded to. For example, a user report of 'Sad' or 'Scared' was more likely to result in a voice call from a friend than a report of 'Happy'. Participants were more likely to respond to an emotional state when logging into the web system than when accessing the mobile system. This may be due to the nature of each system. For participants to access the web system, they must go to the application, sign in, and carry out a task (check friends states, update their own, and perhaps respond to the states of others). In contrast, the mobile application offers an ad-hoc experience in which one can tap an icon and see a list of states, and make a choice based on those states. One participant noted that the opportunity for interaction with the system was greater with the mobile application than the web based system because of the serendipitous nature of a mobile application.

Emotion report behaviours remained balanced across both mediums, except for the frequency of report of the 'Scared' state. This suggests that while the platform may not influence use, the context in which the technology is used may be allowing for a broader report of emotion state. Desktop and web based systems confine their users to situations in which changes to emotional state may occur less frequently and with less variance. For example, having access to the system solely through a website may prevent a user from sharing their emotional state in situations where web access is less convenient (such as out with friends or on a bus). The mobile system however, allowed users to interact with the system at any time and in any location. This resulted in the user reporting emotion that may have otherwise have been missed in a web-based system.

Participants reported that they were more aware of the emotional states of others when using the Emotishare system. Reponses to the surveys and focus group indicated that participants were using the system to help them make decisions around communicating with others in the group. 
Reports from participants that they were more comfortable to share their emotional state via their mobile phone may also have had an impact on these results. For example, a participant may have been more willing to report a stronger emotion (such as 'Scared') using their mobile phone, than using a web interface. This suggests that the context of a native mobile application sitting alongside their own personal data may have an impact on how a user perceives the security and privacy of their data. In addition, the mobile device is more commonly used for communication of personal data (such as SMS and voice messages) and would therefore be less of a change from existing user behaviour with these types of devices.

Group types are one factor found to contribute to the success of the Emotishare system. Participants indicated that they were more comfortable to share this type of information with smaller groups and also suggested that they would consider the emotion reports more truthful and indicative of actual emotional state when viewed from this type of system. These intimacy groups (as defined by: Lickel et al., 2000) are not well supported in current social networking systems. This research suggests how we can better support emotional communication between groups, and identifies those groups that may best benefit from this type of technology (e.g. smaller more intimate group types).

Overall, these results suggest that mobile systems provide more opportunities for emotion communication between groups than web-based systems. While both systems showed similar usage patterns with regards to the emotions reported and responded to, the accessibility of the mobile devices and the reported personal attachment of the participants to these devices made them a better candidate for system use.

\section{FUTURE WORK}

Future research could investigate further the types of groups best supported by this type of system. This research has indicated that smaller and more intimate groups would benefit from a system such as Emotishare, however opportunities still exist to further refine the attributes of these groups. For example, groups geographically dispersed or support networks dealing with depression or autism may also benefit from this system.

This research encourages further investigation of the expression of emotion state within and between groups. For example, Fineman (2003, 2008) suggests that people appropriate emotional states based on the relationship they have with the person with whom they are expressing the emotion. The
Emotishare system allows researchers to record and monitor the emotional state of individuals as they interact with the system, and map responses and relationships as they are formed in the system.

\section{ACKNOWLEDGMENTS}

The authors would like to thank the mother's groups who participated in this research project.

\section{REFERENCES}

Coupland, N., Giles, H. \& Wiemann, J. (1991) "Miscommunication" and problematic talk, Sage Publications, London.

Derks, D., Fischer, A. H. \& Bos, A. E. R. (2008) The role of emotion in computer-mediated communication: A review. Computers in Human Behavior, 24, 766-785.

Facebook. (2012) Facebook

http://www.facebook.com (January, 2012).

Fineman, S. (2003) Understanding emotion at work, SAGE Publications, London.

Fineman, S. (2008) The emotional organization: passions and power, Blackwell Pub, MA, USA.

Kato, Y., Kato, S. \& Akahori, K. (2007) Effects of emotional cues transmitted in e-mail communication on the emotions experienced by senders and receivers. Computers in Human Behavior, 23, 1894-1905.

Lickel, B., Hamilton, D. L., Wieczorkowska, G., Lewis, A., Sherman, S. J. \& Uhles, A. N. (2000) Varieties of groups and the perception of group entitativity. Journal of Personality and Social Psychology, 78, 223-246.

Lo, S.-K. (2008) The Nonverbal Communication Functions of Emoticons in Computer-Mediated Communication. CyberPsychology and Behaviour, 11, 595-597.

Planalp, S. (1999). Communicating emotion: social, moral, and cultural processes, Cambridge University Press, Cambridge.

Planalp, S. \& Knie, K. (2002) Integrating Verbal and Nonverbal Emotion(al) Messages. In Fussell, S. R. (ed.) The verbal communication of emotions: interdisciplinary perspectives. L. Erlbaum Associates, NJ, USA.

Plutchik, R. (1980) Emotion: A Psychoevolutionary Synthesis, Harper \& Row, New York.

Riordan, M. A. \& Kreuz, R. J. (2010) Cues in computer-mediated communication: $A$ corpus analysis. Computers in Human Behavior, 26, 18061817.

Twitter. (2012) Twitter http://www.twitter.com (January, 2012).

Willis, M. J. (2011) Emotishare: Enabling Computer Mediated Emotion Communication Through Networked Devices. PhD, University of the Sunshine Coast, Australia. 